\title{
Osteoprotegerin Predicts Long-Term Outcome in Patients with ST-Segment Elevation Myocardial Infarction Treated with Primary Percutaneous Coronary Intervention
}

\author{
Sune Pedersen $^{a} \quad$ Rasmus Mogelvang ${ }^{d}$ Mette Bjerre $^{c} \quad$ Jan Frystyk ${ }^{c}$ \\ Allan Flyvbjerg $^{c}$ Soren Galatius ${ }^{a}$ Tor Biering Sørensen ${ }^{a} \quad$ Allan Iversen $^{a}$ \\ Anders Hvelplund $^{\mathrm{a}}$ Jan S. Jensen ${ }^{\mathrm{a}, \mathrm{b}}$ \\ ${ }^{\mathrm{a}}$ Department of Cardiology P, Copenhagen University Hospital, and ${ }^{\mathrm{b}} \mathrm{Clinical}$ Institute of Surgery and Internal \\ Medicine, Faculty of Health Sciences, University of Copenhagen, Copenhagen, 'Department of Endocrinology and \\ Internal Medicine, Medical Research Laboratories, Clinical Institute of Medicine, Aarhus Faculty of Health Sciences \\ and Aarhus University Hospital, Aarhus, and ' Department of Medicine, Holbæk Hospital, Holbæk, Denmark
}

\section{Key Words}

Inflammation - Myocardial infarction - Osteoprotegerin •

Percutaneous coronary intervention $\cdot$ ST-segment

elevation · STEMI

\begin{abstract}
Background: Osteoprotegerin (OPG) is a glycoprotein with a regulatory role in immune, skeletal and vascular systems. Data suggest that high circulating OPG levels are associated with an increased risk of cardiovascular disease. We analyzed the association between OPG and long-term outcome in patients with ST-segment elevation myocardial infarction (STEMI) treated with primary percutaneous coronary intervention ( $\mathrm{pPCl}$ ). Methods: We included 716 consecutive STEMI patients admitted to a single high-volume invasive heart center from September 2006 to December 2008. Endpoints were all-cause mortality, repeat myocardial infarction, admission due to heart failure and combinations thereof. Median follow-up lasted 27 months (interquartile range: 22-33). Results: OPG levels exhibited a non-Gaussian distribution and were therefore divided into quartiles. High levels of OPG
\end{abstract}

were significantly associated with a worse outcome. After adjustment for conventional risk factors (e.g. C-reactive protein, estimated glomerular filtration rate, symptom-to-balloon time and troponin I) using Cox regression, OPG remained a significantly independent predictor of death (HR per increase in OPG quartile: $1.28 ; \mathrm{Cl}: 1.03-1.59 ; \mathrm{p}=0.03)$, repeat myocardial infarction (HR: $1.30 ; \mathrm{Cl}: 1.00-1.68 ; \mathrm{p}=0.05$ ) and admission with heart failure (HR: 1.50; Cl: 1.18-1.90; $\mathrm{p}=$ 0.001). Conclusion: This study shows that OPG independently predicts long-term outcome in STEMI patients treated with $\mathrm{pPCl}$. Eventually, this knowledge could improve risk stratification and overall outcome.

Copyright $\odot 2012$ S. Karger AG, Basel

\section{Introduction}

Coronary artery disease (CAD) is the leading cause of death and coronary heart failure in industrialized countries and causes decreases in quality of life as well as increases in health care burdens. Numerous efforts have been made to improve the prognosis in these patients. It

\section{KARGER}

Fax +4161306 1234 E-Mail karger@karger.ch www.karger.com
(C) 2012 S. Karger AG, Basel

$0008-6312 / 12 / 1231-0031 \$ 38.00 / 0$

Accessible online at:

www.karger.com/crd
Sune Haahr Pedersen, MD

Department of Cardiology P, Gentofte University Hospital

Niels Andersens Vej 65

DK-2900 Copenhagen (Denmark)

Tel. +45 3022 0889, E-Mail peidersen@ hotmail.com 
is now well established that the most effective treatment strategy of acute myocardial infarction (MI) is immediate reperfusion by primary percutaneous coronary intervention (pPCI) or thrombolytic agents $[1,2]$. Nevertheless, patients surviving an acute MI have a $10 \%$ risk of mortality and a $25 \%$ risk of cardiac failure within 1 year [3]. Therefore, efforts to improve risk stratification, clarify the pathophysiological mechanisms and identify targets for therapeutic interventions should be done. One challenge is to elucidate why otherwise comparable MI patients (e.g. similar age, diabetes status and treatment delay) end up with very different outcomes. Apparently, conventional risk factors are inadequate in predicting adverse outcome and consequently increasing attention is directed towards improving risk stratification. A number of biomarkers have been associated with ischemia, inflammation, atherosclerosis and myocardial damage [47]. However, currently only cardiac-specific troponins [troponin $\mathrm{T}(\mathrm{TnT})$ and troponin I (TnI)] and creatinekinase $\mathrm{MB}(\mathrm{CK}-\mathrm{MB})$ are being used for diagnosis and - to some extent - risk stratification in patients with acute coronary syndromes.

Growing evidence suggests that inflammatory pathways influence the course of CAD, both with regard to the development of mild CAD, but also to outcome in patients with established severe CAD due to plaque destabilization and susceptibility to ischemia-driven myocardial damage [8-11].

Osteoprotegerin (OPG) is a glycoprotein which serves as a soluble receptor for two members of the tumor necrosis factor receptor superfamily - nuclear factor $\kappa \mathrm{B}$ ligand (RANKL) and tumor necrosis factor-related apoptosis-inducing ligand (TRAIL) [12]. OPG is found in many different tissues, including bones, arteries and plasma. Current knowledge indicates that OPG plays not only an important role in the development of skeletal disorders but also in the course of cardiovascular diseases due to its proinflammatory effects [13]. In the general population, high plasma levels of OPG have been found to increase the risk of coronary artery calcium and coronary events, impaired left ventricular function and cardiovascular death [14-17]. In patients with established severe ischemic disease such as acute coronary syndromes, elevated plasma OPG has also been associated with an increased risk of impaired outcome [18-20]. The negative effect of OPG is likely caused by an increased migration of lymphocytes and neutrophils to the intimal layer of the vessel wall, which in turn increases the risk of atherosclerosis [21]. Furthermore, high plasma OPG reduces the thickness of the fibrous cap surrounding the athero- sclerotic plaque by up-regulation of matrix metalloproteinase 9. This may eventually lead to plaque rupture, thrombus formation and MI [22-25] (fig. 1). In addition, as increased plasma OPG induces a higher degree of inflammation [26], this could lead to an increased risk of ischemia/reperfusion injury and, consequently, ST-segment elevation MI (STEMI) patients with high OPG levels undergoing rapid reperfusion during pPCI face a potential increased risk of ischemia/reperfusion injury and thus myocardial damage [27]. These OPG-induced mechanisms may eventually translate into an increased risk of heart failure, MI and death.

The role of OPG as a prognostic biomarker in STEMI patients treated with PPCI is unclear, and improved risk stratification in this subset of patients could eventually lead to an improved overall outcome. Consequently, we analyzed OPG in the plasma of STEMI patients treated with PPCI and evaluated its potential prognostic value.

\section{Methods}

Study Population

Routine treatment with pPCI for STEMI was introduced and implemented in Denmark in 2002 following the presentation of the DANAMI-2 trial at the American College of Cardiology Meeting in 2002 [1]. The Gentofte University Hospital has a pPCI catchment population of 1.2 million citizens, i.e. $>20 \%$ of the total Danish population, and is the invasive hub for 10 noninvasive centers, serving a predominantly urban population. On-site cardiac surgery is present and $>1,500$ PCI procedures are performed annually (approx. 500 as pPCI), with each individual PCI operator performing approximately $300 \mathrm{PCI}$ and 100 pPCI per year (mean: 89 pPCI per year; median: 99 pPCI per year, range: 11-138 pPCI).

From September 2006 to December 2008, a total of 716 STEMI patients were admitted and treated with pPCI and plasma samples were analyzed for OPG. In the instance of $>1$ pPCI procedure within this period, the first procedure was defined as the index procedure. Inclusion criteria and definition of STEMI was: presence of chest pain for $>30$ min and $<12 \mathrm{~h}$ and cumulative persistent ST-segment elevation $\geq 4 \mathrm{~mm}$ in at least 2 contiguous precordial ECG leads or $\geq 2 \mathrm{~mm}$ in at least 2 contiguous limb ECG leads [a limited number $(\mathrm{n}=10)$ of patients were triaged to PPCI due to newly developed left bundle branch block]. Furthermore, a significant increase in $\mathrm{TnI}(>0.40 \mu \mathrm{g} / \mathrm{l})$ was required for inclusion in the present study [TnI kit: the commercially available Siemens Healthcare, Diagnostics Immulite 2500, STAT-Troponin I (ref $<0.40 \mu \mathrm{g} / \mathrm{l})]$.

\section{Baseline and Procedural Data}

The baseline data were prospectively collected from all patients and entered in a dedicated registry. Hypertension was defined as use of blood pressure-lowering drugs on admission. Diabetes was defined as use of glucose-lowering drugs on admission, fasting plasma glucose concentration $\geq 7 \mathrm{mmol} / \mathrm{l}$ or non-fasting 


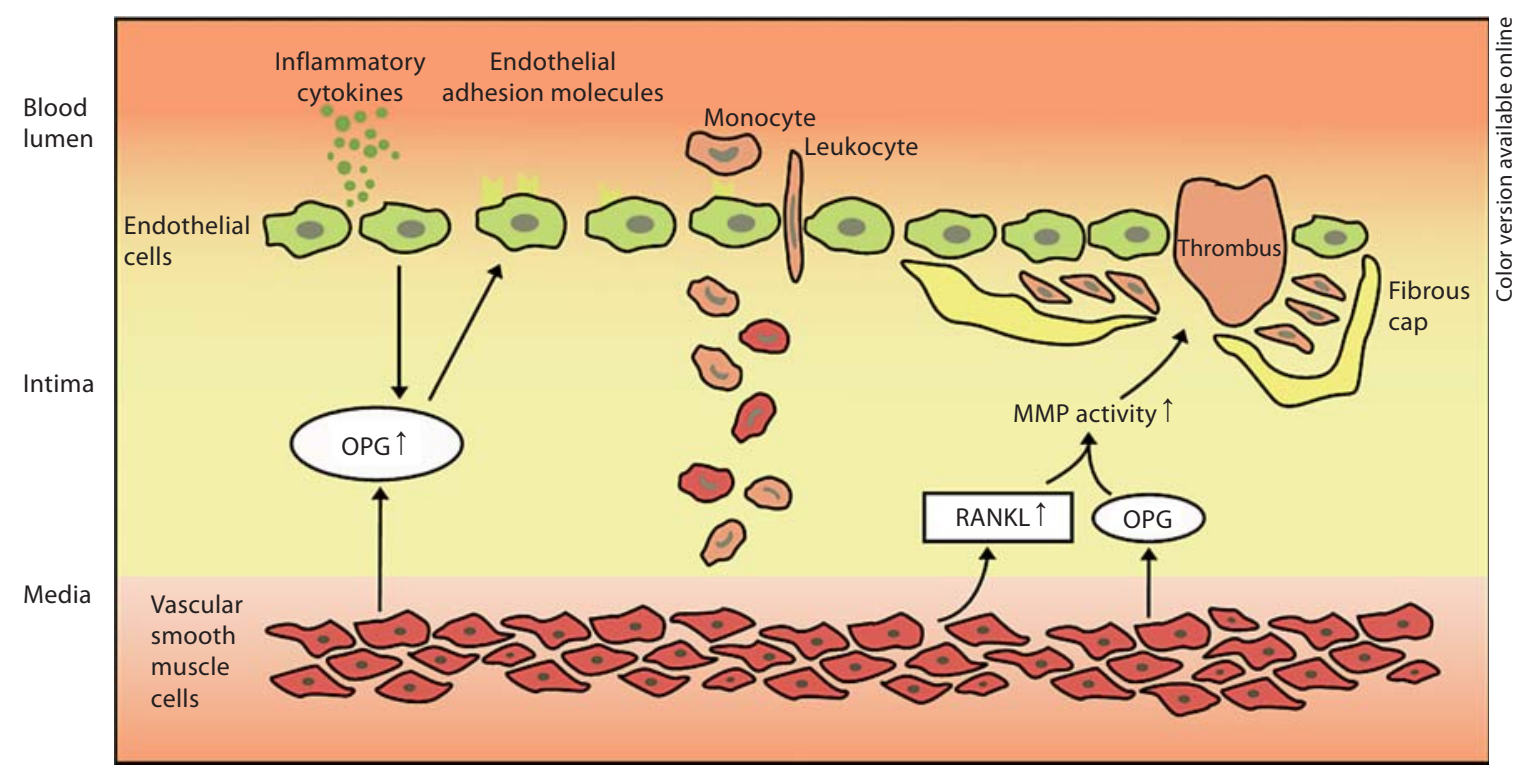

Fig. 1. Increasing OPG levels leading to atherosclerosis. Increased expression of inflammatory cytokines close to the endothelial cells leads to up-regulation of OPG expression and endothelial cell adhesion molecules. The adhesion molecules facilitate entering of monocytes and leukocytes to the intimal layer of the vessel wall. The monocytes and leukocytes enhance the expression of
RANKL and the formation of vascular smooth muscle cells. RANKL and OPG promote the activity of matrix metalloproteinase, which results in the degradation of the extracellular matrix. This degradation leaves the fibrous cap thinner and more fragile, which can lead to thrombus formation and MI. MMP = Matrix metalloproteinase. plasma glucose concentration $\geq 11.1 \mathrm{mmol} / \mathrm{l}$. Multivessel disease was defined as 2- or 3-vessel disease and complex lesions such as type $\mathrm{C}$ lesions.

TnI levels were determined on admission, and 6 and $12 \mathrm{~h}$ after admission. Peak TnI was used in the statistical analyses divided into quartiles. C-reactive protein (CRP) and estimated glomerular filtration rate (eGFR) were also assessed on admission.

STEMI patients treated with pPCI at our institution are admitted from several primary hospitals, and post-PCI treatment is performed at these hospitals in $\sim 50 \%$ of all cases. At our institution, echocardiography is performed during the initial 5-day period of admission, while other institutions have other protocols. In order to present a homogeneous estimate of left ventricular ejection fraction (LVEF), only patients with echocardiography performed within 5 days after STEMI were included in the echosubstudy. Echocardiography was performed using GE Vivid 7 and 9, 1-5 days after MI [median: 2, interquartile range (IQR): 1-3]. LVEF was 'eyeballed by experts' guided by wall motion index.

\section{pPCI Procedure}

Outside normal office hours, the catheterization laboratory staff was activated from their homes ( $<30 \mathrm{~min}$ away) by the cardiologist at the invasive center who had performed the triage for pPCI. During office hours, the crew was present at the catheterization laboratory. pPCI was performed according to contemporary interventional guidelines and 10,000 IU of unfractionated heparin, $300 \mathrm{mg}$ acetyl salicylic acid and $600 \mathrm{mg}$ clopidogrel were given preoperatively. Glycoprotein IIb/IIIa inhibitors were used at the discretion of the operator. The transfemoral approach was used with 6- or 8-french sheaths, conventional devices and Iomeron contrast fluid (Bracco, Milan, Italy). Subsequent medical treatment included anti-ischemic, lipid-lowering and anti-thrombotic drugs according to current treatment guidelines. Importantly, at our institution, all STEMI patients treated with pPCI after January 2004 were discharged with a prescription of a 75-mg daily dose of clopidogrel for 12 months - regardless of the stent type implanted.

\section{Measurement of Plasma OPG}

Peripheral arterial blood was drawn from the femoral sheath at the beginning of the procedure, thus avoiding contamination with contrast fluid. Blood was allocated to different containers including 4-ml EDTA containers and was centrifuged at 10,000 rpm for 10 min within 0.5 h. Plasma was stored in NuncCryo tubes (Nunc, Roskilde, Denmark) at $-80^{\circ} \mathrm{C}$.

Plasma levels of OPG were quantified using commercially available monoclonal antibodies (DY085E; R\&D Systems, Abingdon, UK) by a time-resolved immunofluorometric assay as previously described [1]. Briefly, wells were coated with $0.1 \mu \mathrm{g}$ antiOPG antibody. Recombinant OPG in the range from 4,000 to $31.25 \mathrm{ng} / \mathrm{l}$ was used as standard and EDTA plasma samples were diluted 4-fold in PBS with 1\% BSA and incubated overnight at $4{ }^{\circ} \mathrm{C}$. Bound OPG was determined by incubation with $20 \mathrm{ng}$ biotinylated anti-OPG detection antibody followed by addition of 10 ng Eu ${ }^{3+}$-labelled streptavidin (Perkin Elmer, Life Sciences, Turku, Finland). Bound europium was detected by the addition of $200 \mu \mathrm{l}$ of enhancement solution and reading the time-resolved fluorescence on a DELFIA fluorometer (Victor ${ }^{3}$; Perkin Elmer). The lim- 
Table 1. Patients divided into quartiles (Q) according to OPG levels

\begin{tabular}{|c|c|c|c|c|c|}
\hline Characteristics & $\begin{array}{l}\text { 1st } \mathrm{Q} \\
763-1,972 \mathrm{ng} / \mathrm{l} \\
(\mathrm{n}=179)\end{array}$ & $\begin{array}{l}\text { 2nd Q } \\
1,973-2,837 \mathrm{ng} / \mathrm{l} \\
(\mathrm{n}=179)\end{array}$ & $\begin{array}{l}3 \mathrm{rd} Q \\
2,838-3,940 \mathrm{ng} / 1 \\
(\mathrm{n}=179)\end{array}$ & $\begin{array}{l}\text { 4th Q } \\
3,941-28,874 \mathrm{ng} / \mathrm{l} \\
(\mathrm{n}=179)\end{array}$ & $\mathrm{p}$ value \\
\hline Mean age $\pm S D$, years & $58.6 \pm 10.3$ & $61.4 \pm 10.8$ & $66.1 \pm 11.6$ & $67.9 \pm 13.2$ & $<0.01$ \\
\hline Males & 84 & 77 & 72 & 63 & $<0.01$ \\
\hline Hypertension & 32 & 32 & 31 & 39 & 0.45 \\
\hline Diabetes & 7 & 6 & 8 & 16 & $<0.01$ \\
\hline Current smokers & 51 & 58 & 50 & 43 & 0.06 \\
\hline Hypercholesterolemia & 21 & 16 & 18 & 22 & 0.35 \\
\hline Previous MI & 7 & 2 & 6 & 8 & 0.04 \\
\hline Known heart failure & 2 & 2 & 2 & 4 & 0.17 \\
\hline Mean BMI $\pm S D$ & $27.3 \pm 4.0$ & $27.2 \pm 4.7$ & $26.1 \pm 4.5$ & $26.2 \pm 4.7$ & 0.01 \\
\hline Peak TnI, $\mu g / 1$ & $89(22-228)$ & $100(35-227)$ & $110(30-267)$ & $89(24-252)$ & 0.63 \\
\hline CRP on admission, $\mathrm{mg} / \mathrm{l}$ & $3(1-5)$ & $3(1-5)$ & $4(1-9)$ & $6(3-18)$ & $<0.01$ \\
\hline eGFR on admission & $76(64-89)$ & $77(62-92)$ & $72(58-85)$ & $67(50-78)$ & $<0.01$ \\
\hline Symptom-to-balloon time, min & $180(120-275)$ & $200(125-310)$ & $195(126-322)$ & $225(149-400)$ & 0.02 \\
\hline LVEF, \% & $40(30-50)$ & $40(30-50)$ & $35(25-50)$ & $35(30-45)$ & 0.34 \\
\hline Multivessel disease & 26 & 25 & 27 & 30 & 0.75 \\
\hline \multicolumn{6}{|l|}{ Lesion type } \\
\hline Type A & 11 & 17 & 8 & 13 & 0.31 \\
\hline Type B & 34 & 37 & 36 & 37 & \\
\hline Type C & 51 & 44 & 52 & 46 & \\
\hline Use of DES & 72 & 74 & 68 & 69 & 0.66 \\
\hline GP IIb/IIIa inhibitors & 34 & 24 & 24 & 22 & 0.06 \\
\hline \multicolumn{6}{|l|}{ Infarct-related artery } \\
\hline LAD & 51 & 39 & 47 & 50 & 0.48 \\
\hline RCA & 37 & 50 & 42 & 49 & \\
\hline $\mathrm{Cx}$ & 11 & 11 & 12 & 10 & \\
\hline $\mathrm{LM}$ & 0 & 0.6 & 0.6 & 0.6 & \\
\hline
\end{tabular}

Data are presented as percentages except where otherwise indicated. $\mathrm{MI}=$ Myocardial infarction; $\mathrm{BMI}=$ body mass index; $\mathrm{TnI}=$ troponin I; eGFR = estimated glomerular filtration rate; LVEF = left ventricular ejection function; DES = drug-elusting stent; GP = glycoprotein. Figures in parentheses are median IQR.

it of detection was $15 \mathrm{ng} / \mathrm{l}$. The intra- and interassay coefficients of variation were below 5 and 9\%, respectively [28].

\section{Follow-Up and Study Endpoints}

Follow-up was $99.7 \%$ complete (2 patients were lost to followup due to emigration). The study endpoints were all-cause mortality, repeat MI and admission with clinical signs of heart failure (dyspnea, fatigue and edema/stasis) combined with a discharge diagnosis of heart failure. Mean follow-up time was 27 months (IQR: 22-33).

Follow-up data on mortality were collected from the National Person Identification Registry, which holds information on vital status (alive, death or emigration). Follow-up data on repeat MI and admission with heart failure were collected using hospital source data, as well as data from the highly validated Danish National Board of Health's National Patient Registry, using ICD10 codes [29]. Repeat MI was defined as recurrent chest pain combined with significant increases in CK-MB $(>5 \mu \mathrm{g} / \mathrm{l})$, TnT $(>0.10 \mu \mathrm{g} / \mathrm{l})$ or TnI $(>0.4 / 0.03 \mu \mathrm{g} / \mathrm{l}$; kit dependent). Thus, both events with non-STEMI and STEMI were classified as repeat MI.

\section{Statistics}

OPG displayed a non-Gaussian distribution and patients were divided into quartiles. Proportions were compared using the $\chi^{2}$ test, continuous Gaussian distributed variables with ANOVA and non-Gaussian distributed variables with the Kruskal-Wallis test. We tested associations between OPG and other baseline variables using univariate logistic or linear regression.

Hazard ratios (HR) were calculated by Cox proportional hazards regression analyses. The assumptions of linearity and proportional hazards in the models were assured. First-order interactions between the OPG variable and other baseline variables for endpoints were assessed in univariate models. In order to maintain a robust model, only 1 variable per 8 events were allowed in the multivariable Cox analyses of each endpoint. Variables with the lowest $p$ value on univariate analysis were included until the maximum allowed number of variables was reached.

In the statistical tests, $\mathrm{p} \leq 0.05$ was considered of statistical significance. SPSS for Windows (version 18.0; SPSS, Chicago, Ill., USA) was used. 


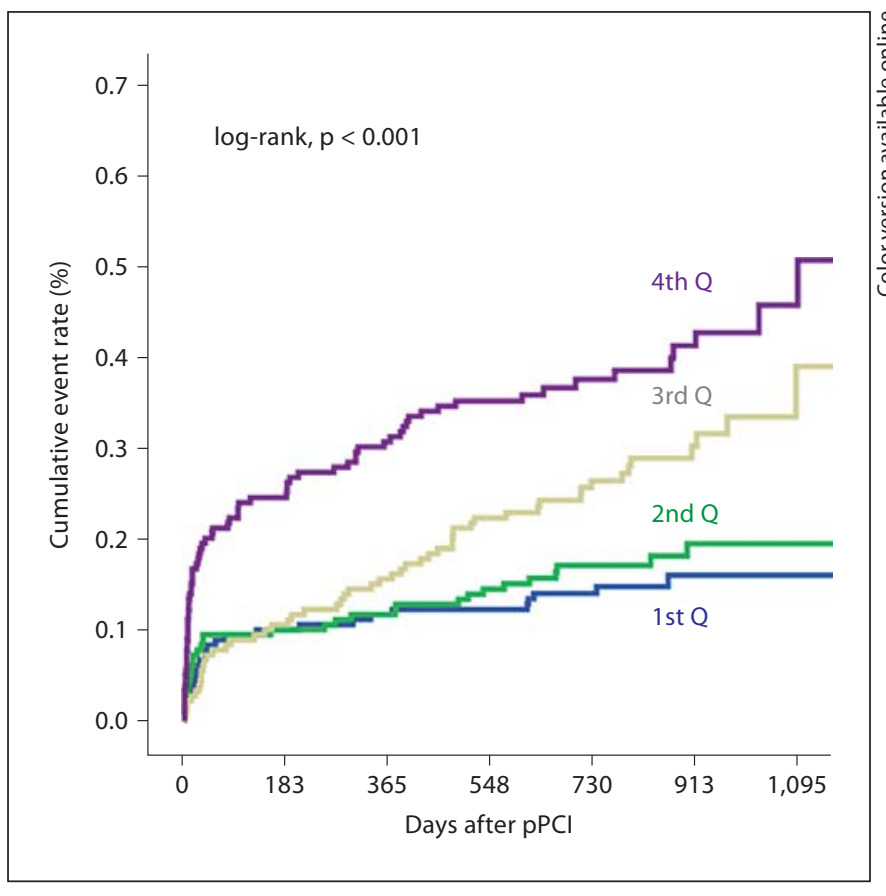

Fig. 2. Unadjusted Kaplan-Meier plots for the combined endpoint of death, repeat MI (non-fatal myocardial re-infarction) and admission due to heart failure according to OPG quartiles (Q); logrank test.

The study was approved by the local scientific ethical committee and the Danish Data Protection Agency, and complied with the Second Declaration of Helsinki.

\section{Results}

During a median follow-up time of 27 months (IQR: 22-33), 93 (13\%) patients died, 51 (7\%) had a repeat MI, 67 (9\%) were admitted with heart failure and $186(26 \%)$ reached the combined endpoint of death, repeat MI or admission with heart failure. Median OPG level was 2,935 ng/l (IQR: 1,973-3,940). High OPG levels were significantly associated with increased age, CRP, eGFR, diabetes, female gender and increased symptom-to-balloon time. No association between peak TnI and OPG was found (table 1).

\section{Long-Term Follow-Up (Complete Cohort, $n=716$ )}

No interactions between OPG and other baseline variables for endpoints were found. The log-rank test based on the Kaplan-Meier curves showed a significant association between high OPG and impaired outcome $(\mathrm{p}<0.001$; fig. 2). This association was confirmed in univariate Cox analysis, where each increase in the OPG quartile led to a $65 \%$ increased risk of dying [HR: $1.65 ; 95 \%$ confidence interval (CI): $1.35-2.01 ; \mathrm{p}<0.001$ ] and a 53\% increased risk of reaching the combined endpoint (HR: 1.53; CI: 1.331.75; $p<0.001)$. Even after adjustment for conventional risk factors, OPG remained a significantly independent predictor of death, repeat MI, admission with heart failure and of the combined endpoint (fig. 3). The incremental prognostic value of OPG is displayed in figure 4 , where $\chi^{2}$ test values of different Cox models are displayed. When peak TnI levels were added to the multivariable Cox analysis (model 2), no significant increase in $\chi^{2}$ test values was found $(p=0.10)$. However, when OPG was added, the $\chi^{2}$ test value significantly increased ( $p<0.001)$, thus confirming the incremental value of OPG (model 3).

\section{Long-Term Follow-Up with Adjustment for LVEF $(n=450)$}

When the variable 'LVEF <45\%' was included in the multivariable Cox analysis, OPG remained a significant predictor of the combined endpoint (HR: 1.34; CI: 1.111.61; $\mathrm{p}<0.001$; adjusted for: age, gender, $\mathrm{LVEF}<45 \%$, diabetes mellitus, previous MI, known heart failure, peak TnI, CRP, eGFR, multivessel disease, complex lesions and symptom-to-balloon time). However, this association was not found in the analyses of the individual endpoints.

\section{Discussion}

In this study, we evaluated the independent impact of OPG on long-term clinical outcome in STEMI patients treated with $\mathrm{pPCI}$ in a real-life setting. After adjustment for conventional risk factors, OPG independently predicted death, repeat MI and admission with heart failure. In a subgroup where baseline LVEF $>45 \%$ was included in the multivariable analyses, OPG still independently predicted impaired outcome.

This study confirms the association between high OPG plasma levels and impaired outcome found in previous studies. In a study by Omland et al. [19], 897 patients with acute coronary syndromes (approx. 1/3 with STEMI, 1/3 with non-STEMI and $1 / 3$ with unstable angina), similar $\mathrm{HR}$ for death and heart failure were reported. In addition, OPG was also found to be a potential marker for cardiovascular mortality and clinical events in patients with MI complicated with heart failure. In a prospective study in 234 patients, high OPG levels at baseline were significant- 
Fig. 3. HR plots based on multivariable Cox analyses. Median follow-up was 2.7 years. Values were adjusted for age, previous MI, complex lesion, CRP and eGFR (all values); diabetes, known heart failure, symptom-to-balloon time (all except for Repeat MI), and gender and peak TnI (Death and Combined), and multivessel disease, LAD lesion, current smoker, body mass index and use of glycoprotein IIb/ IIIa inhibitor (Combined).

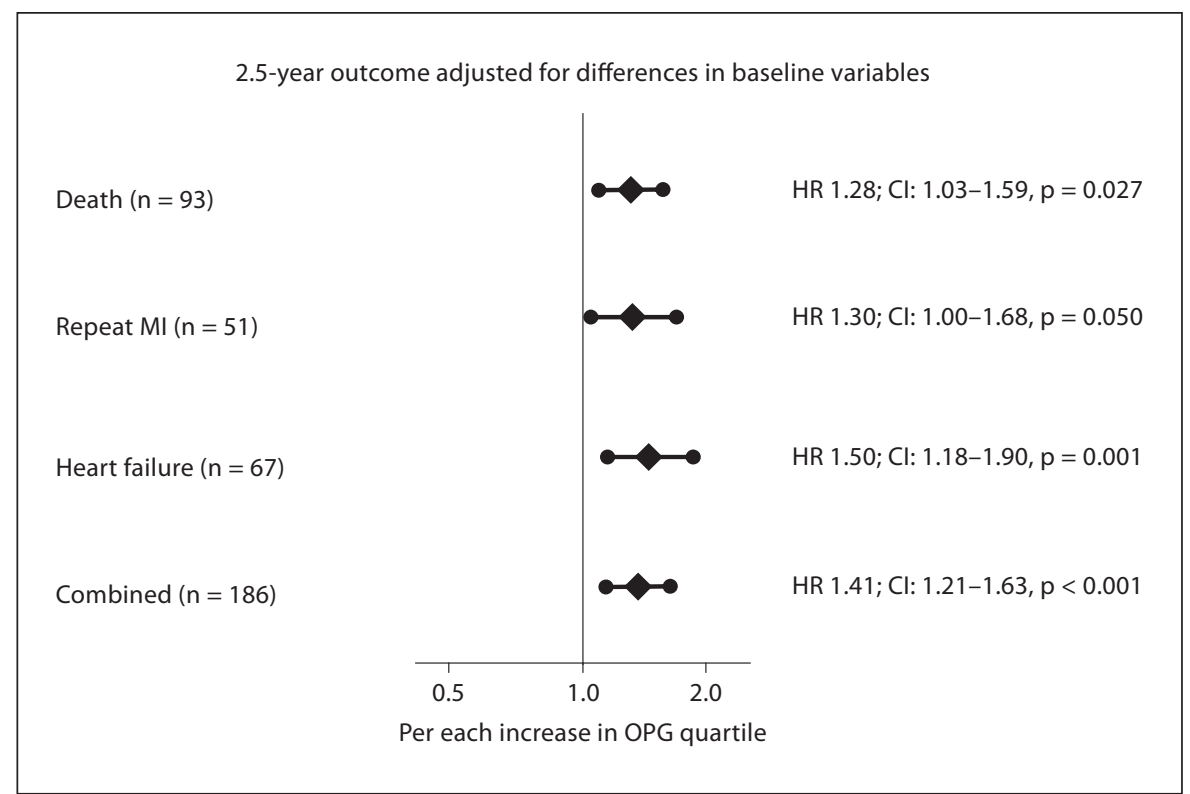

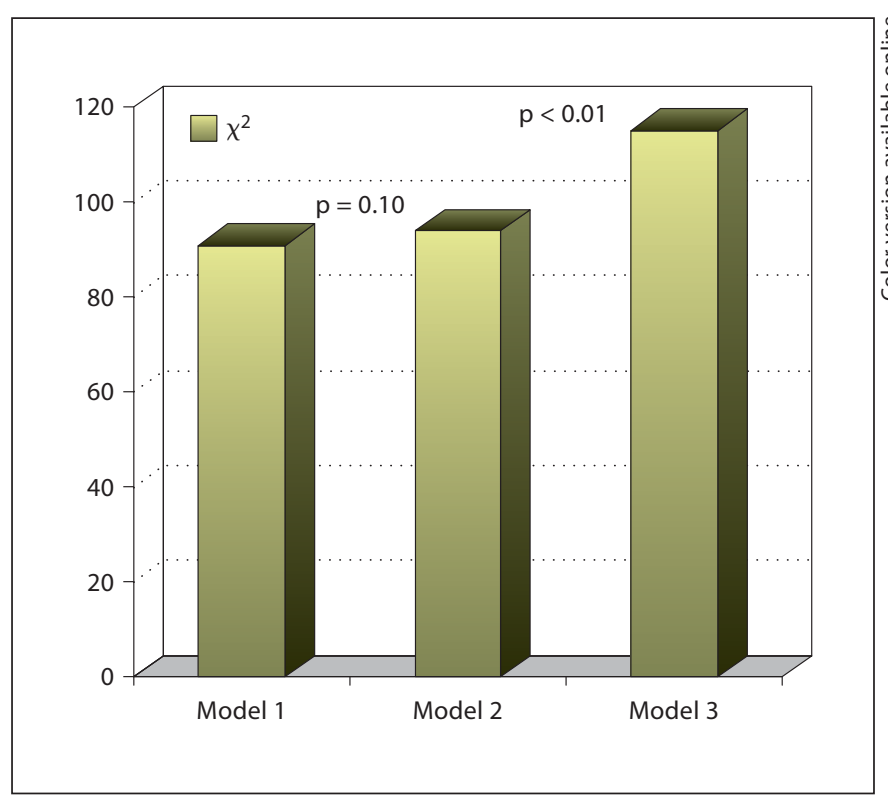

Fig. 4. Incremental value of OPG in predicting the combined endpoint: death, repeat MI or admission with heart failure. Model 1 includes age, gender, hypertension, diabetes mellitus, previous MI, known heart failure, peak TnI, symptom-to-balloon time, multivessel disease, complex lesion, glycoprotein IIb/IIIa inhibitor use, LAD lesion, body mass index and smoking. Model 2 includes Model $1+$ peak TnI (divided into quartiles). Model 3 includes Model $1+$ peak TnI and OPG. ly associated with increased risk of cardiovascular death and all-cause mortality [18]. Recently, Andersen et al. [20] have demonstrated in 199 STEMI patients that OPG levels above the median were associated with a larger infarct size at 3 months measured by SPECT imaging.

The important information obtained from the present study is that OPG may have the potential to improve risk stratification and thus guide treatment/observation strategies in a large high-risk CAD population consisting entirely of STEMI patients treated with pPCI according to current guidelines. Only a minor proportion of our patients had a previous MI or known heart failure prior to their STEMI, and our main results were adjusted for a large number of potential confounders. These circumstances add to the importance of our findings, as they provide a very clear message regarding OPG as an independent predictor of impaired outcome.

Furthermore, this study confirms findings from previous studies showing that high levels of OPG are association with increased age, diabetes and female gender $[19,30,31]$. If a clinical relevant cutoff point of OPG is to be implemented, these associations need to be taken into account, and studies of OPG in the general population preferable using similar OPG-kits - would add valuable information.

Somewhat surprisingly, we could not find an association between OPG and peak TnI, and more interestingly no association between peak TnI and outcome was found. Even though troponin could be regarded as marker of 
myocardial damage and thus heart failure and consequent mortality, studies of troponin as a prognostic marker have been conflicting. A number of studies have found a significant association between high troponin levels and impaired outcome [32-34]. However, other studies have clearly failed to demonstrate this association. In a study of 207 STEMI patients treated with pPCI, patients with high TnI levels had a $2 \%$ increased risk of heart failure at 1 year (HR: 1.02; CI: 1.00-1.03), but high TnI levels were unable to predict 1-year mortality or reinfarction [35]. In a similar but larger study $(n=1,237)$ of STEMI patients treated with pPCI, high TnT showed no association with 1-year mortality $(p=0.31)[36]$. One obvious reason for this lack of association could relate to the time-dependent excretion of troponin during MI. In patients with long 'symptom-toballoon' time (and thus expected poor outcome), peak troponin may not have been measured. Furthermore, in animal models it has been shown that rapid reperfusion (within $2 \mathrm{~h}$ ) increases the amount of enzyme released from the myocytes to the plasma compared to delayed reperfusion [37]. If a similar effect is present in STEMI patients, it could partly explain the poor prognostic value of troponin.

In general practice, echocardiography is a valuable tool in risk stratification, and patients with LVEF $<45 \%$ are usually treated with angiotensin-converting enzyme inhibitors and stratified to extensive follow-up in heart failure clinics. On the other hand, patients with a 'lowrisk' profile are less likely to receive extensive treatment and close clinical control after STEMI. In our subgroup analyses where LVEF $<45 \%$ was included in the multivariable analysis, OPG remained a significant independent predictor of the combined endpoint: death, repeat MI or admission with heart failure. This suggests that a risk stratification partly based on OPG levels may improve long-term outcome by optimizing clinical control, treatment and focus on patient compliance.

\section{Study Limitations}

Despite our efforts to reduce bias, the risk of unknown confounders exists in a non-randomized trial (residual confounding). We found a relatively low prevalence of diabetes, previous MI and known heart failure prior to STEMI. Validation of both baseline variables and endpoints was done in highly validated registries and in-hospital source data. However, the number of endpoints may have been slightly underestimated, although it is likely that this potential underestimation was equally distributed in all the OPG groups. The lower fraction of diabetes in our population could - to some extent - be explained by a lower background prevalence of diabetes in Den- mark compared to the US for example. We used TnI (and in $2 / 3 \mathrm{LVEF}$ ) as surrogate markers for infarct size. It would have been preferable to have a more precise measurement such as magnetic resonance imaging. Care should be taken though, if the results are to be extrapolated to populations with very different distributions of potential risk factors and logistic facilities. Finally, our geographical and organizational conditions may not necessarily apply to other countries and regions, and our findings should not be extrapolated to settings without high-volume PCI centers.

\section{Perspectives}

In recent years, a large number of biomarkers have been examined in order to predict outcome and consequently improve patient care. Biomarkers have the obvious advantage of being relatively cheap and easy to obtain compared to more extensive and invasive diagnostic imaging procedures. Apart from providing a diagnostic tool, biomarker evaluation also provides valuable knowledge of pathophysiological mechanisms and potential targets for intervention.

Even though the association between cardiovascular disease and high OPG levels seems apparent, the cause of this association is still poorly understood and future studies need to further clarify the pathophysiological mechanisms - especially since some in vitro studies and animal studies have provided results conflicting with the findings from large human studies. If OPG is to be implemented as a prognostic biomarker, it is furthermore required that cutoff values are established. We believe that OPG is associated with CAD and that this biomarker eventually could be a valuable tool in the risk stratification of patients with different degrees of CAD.

\section{Conclusions}

After adjustment for conventional risk factors, OPG independently predicted death, repeat $\mathrm{MI}$ and admission with heart failure in STEMI patients treated with pPCI. In a subgroup where baseline LVEF was included in the multivariable analyses, OPG still independently predicted impaired outcome. Measurement of OPG may be useful in risk stratification and thus improve outcome in a high-risk STEMI population.

\section{Conflict of Interest}

None declared. 


\section{References}

-1 Andersen HR, Nielsen TT, Rasmussen K, Thuesen L, Kelbæk H, Thayssen P, Abildgaard U, Pedersen F, Madsen JK, Grande P, Villadsen AB, Krusell LR, Haghfelt T, Lomholt P, Husted SE, Vigholt E, Kjærgard HK, Mortensen LS: A comparison of coronary angioplasty with fibrinolytic therapy in acute myocardial infarction. $\mathrm{N}$ Engl J Med 2003;349:733-742.

$\checkmark 2$ Keeley EC, Hillis LD: Primary PCI for myocardial infarction with ST-segment elevation. N Engl J Med 2007;356:47-54.

3 Keeley EC, Boura JA, Grines CL: Primary angioplasty versus intravenous thrombolytic therapy for acute myocardial infarction: a quantitative review of 23 randomised trials. Lancet 2003;361:13-20.

-4 Scirica BM: Acute coronary syndrome: emerging tools for diagnosis and risk assessment. J Am Coll Cardiol 2010;55:1403-1415.

5 Gupta S, Drazner MH, de Lemos JA: Newer biomarkers in heart failure. Heart Fail Clin 2009;5:579-588.

-6 Niccoli G, Montone RA, Ferrante G, Crea F: The evolving role of inflammatory biomarkers in risk assessment after stent implantation. J Am Coll Cardiol 2010;56:1783-1793.

-7 Jeong YH, Lee SW, Lee CW, Hong MK, Kim JJ, Park SW, Park SJ, Park DW, Kim YH: Biomarkers on admission for the prediction of cardiovascular events after primary stenting in patients with ST-elevation myocardial infarction. Clin Cardiol 2008;31:572-579.

8 Hansson GK: Inflammation, atherosclerosis, and coronary artery disease. N Engl J Med 2005;352:1685-1695.

9 Libby P, Ridker PM, Hansson GK: Inflammation in atherosclerosis: from pathophysiology to practice. J Am Coll Cardiol 2009;54: 2129-2138.

10 Yellon DM, Hausenloy DJ: Myocardial reperfusion injury. N Engl J Med 2007;357: 1121-1135.

-11 Arumugam TV, Magnus T, Woodruff TM, Proctor LM, Shiels IA, Taylor SM: Complement mediators in ischemia-reperfusion injury. Clin Chim Acta 2006;374:33-45.

12 Flyvbjerg A: Diabetic angiopathy, the complement system and the tumor necrosis factor superfamily. Nat Rev Endocrinol 2010;6 94-101.

13 Reid P, Holen I: Pathophysiological roles of osteoprotegerin (OPG). Eur J Cell Biol 2009; 88:1-17.

14 Abedin M, Omland T, Ueland T, Khera A, Aukrust P, Murphy SA, Jain T, Gruntmanis U, McGuire DK, de Lemos JA: Relation of osteoprotegerin to coronary calcium and aortic plaque (from the Dallas Heart Study). Am J Cardiol 2007;99:513-518.

-15 Omland T, Drazner MH, Ueland T, Abedin M, Murphy SA, Aukrust P, de Lemos JA: Plasma osteoprotegerin levels in the general population: relation to indices of left ventricular structure and function. Hypertension 2007;49:1392-1398.
16 Kiechl S, Schett G, Wenning G, Redlich K, Oberhollenzer M, Mayr A, Santer P, Smolen J, Poewe W, Willeit J: Osteoprotegerin is a risk factor for progressive atherosclerosis and cardiovascular disease. Circulation 2004;109:2175-2180.

17 Semb AG, Ueland T, Aukrust P, Wareham NJ, Luben R, Gullestad L, Kastelein JJ, Khaw KT, Boekholdt SM: Osteoprotegerin and soluble receptor activator of nuclear factor-kappaB ligand and risk for coronary events: a nested case-control approach in the prospective EPIC-Norfolk population study 1993-2003. Arterioscler Thromb Vasc Biol 2009;29:975-980.

18 Ueland T, Jemtland R, Godang K, Kjekshus J, Hognestad A, Omland T, Squire IB, Gullestad L, Bollerslev J, Dickstein K, Aukrust P: Prognostic value of osteoprotegerin in heart failure after acute myocardial infarction. J Am Coll Cardiol 2004;44:1970-1976.

19 Omland T, Ueland T, Jansson AM, Persson A, Karlsson T, Smith C, Herlitz J, Aukrust P, Hartford M, Caidahl K: Circulating osteoprotegerin levels and long-term prognosis in patients with acute coronary syndromes. J Am Coll Cardiol 2008;51:627-633.

20 Andersen GO, Knudsen EC, Aukrust P, Yndestad A, Oie E, Muller C, Seljeflot I, Ueland $\mathrm{T}$ : Elevated serum osteoprotegerin levels measured early after acute ST-elevation myocardial infarction predict final infarct size. Heart 2011;97:460-465.

21 Tsirpanlis G: Is inflammation the link between atherosclerosis and vascular calcification in chronic kidney disease? Blood Purif 2007;25:179-182.

22 Shaker OG, El-Shehaby A, Nabih M: Possible role of osteoprotegerin and tumor necrosis factor-related apoptosis-inducing ligand as markers of plaque instability in coronary artery disease. Angiology 2010;61:756-762.

23 Newby AC: Metalloproteinases and vulnerable atherosclerotic plaques. Trends Cardiovasc Med 2007;17:253-258.

24 Molloy KJ, Thompson MM, Jones JL, Schwalbe EC, Bell PR, Naylor AR, Loftus IM: Unstable carotid plaques exhibit raised matrix metalloproteinase- 8 activity. Circulation 2004;110:337-343.

25 Montecucco F, Steffens S, Mach F: The immune response is involved in atherosclerotic plaque calcification: could the RANKL/ RANK/OPG system be a marker of plaque instability? Clin Dev Immunol 2007;2007: 75805 .

26 Sandberg WJ, Yndestad A, Oie E, Smith C, Ueland T, Ovchinnikova O, Robertson AK, Muller F, Semb AG, Scholz H, Andreassen AK, Gullestad L, Damas JK, Froland SS, Hansson GK, Halvorsen B, Aukrust P: Enhanced T-cell expression of RANK ligand in acute coronary syndrome: possible role in plaque destabilization. Arterioscler Thromb Vasc Biol 2006;26:857-863.

-27 Arslan F, de Kleijn DP, Timmers L, Doevendans PA, Pasterkamp G: Bridging innate im- munity and myocardial ischemia/reperfusion injury: the search for therapeutic targets. Curr Pharm Des 2008;14:1205-1216.

28 Rasmussen LM, Tarnow L, Hansen TK, Parving HH, Flyvbjerg A: Plasma osteoprotegerin levels are associated with glycaemic status, systolic blood pressure, kidney function and cardiovascular morbidity in type 1 diabetic patients. Eur J Endocrinol 2006;154:75-81.

29 Madsen M, Davidsen M, Rasmussen S, Abildstrom SZ, Osler M: The validity of the diagnosis of acute myocardial infarction in routine statistics: a comparison of mortality and hospital discharge data with the Danish MONICA registry. J Clin Epidemiol 2003;56: 124-130.

30 Nybo M, Rasmussen LM: The capability of plasma osteoprotegerin as a predictor of cardiovascular disease: a systematic literature review. Eur J Endocrinol 2008;159:603-608.

- 31 Jono S, Otsuki S, Higashikuni Y, Shioi A, Mori K, Hara K, Hashimoto H, Ikari Y: Serum osteoprotegerin levels and long-term prognosis in subjects with stable coronary artery disease. J Thromb Haemost 2010;8: $1170-1175$.

32 Sherwood MW, Morrow DA, Scirica BM, Jiang S, Bode C, Rifai N, Gerszten RE, Gibson CM, Cannon CP, Braunwald E, Sabatine MS: Early dynamic risk stratification with baseline troponin levels and 90-minute ST-segment resolution to predict 30-day cardiovascular mortality in ST-segment elevation myocardial infarction: analysis from CLopidogrel as Adjunctive ReperfusIon TherapY (CLARITY)-Thrombolysis in Myocardial Infarction (TIMI) 28. Am Heart J 2010;159: 964-971.

33 Chiostri M, Valente S, Crudeli E, Giglioli C, Gensini GF: A new post-PCI scoring system for in-hospital mortality in STEMI patients. J Cardiovasc Med (Hagerstown) 2010;11: 733-738.

- 34 Napan S, Kashinath RC, Kadri S, Orig MN, Khadra S: Prognostic significance of preprocedural troponin-I in patients with non-ST elevation acute coronary syndromes undergoing percutaneous coronary intervention. Coron Artery Dis 2010;21:261-265.

35 Jeong YH, Lee SW, Lee CW, Hong MK, Kim JJ, Park SW, Park SJ, Park DW, Kim YH: Biomarkers on admission for the prediction of cardiovascular events after primary stenting in patients with ST-elevation myocardial infarction. Clin Cardiol 2008;31:572-579.

36 Byrne RA, Ndrepepa G, Braun S, Tiroch K, Mehilli J, Schulz S, Schomig A, Kastrati A: Peak cardiac troponin-T level, scintigraphic myocardial infarct size and one-year prognosis in patients undergoing primary percutaneous coronary intervention for acute myocardial infarction. Am J Cardiol 2010; 106:1212-1217.

- 37 Gibbons RJ, Valeti US, Araoz PA, Jaffe AS: The quantification of infarct size. J Am Coll Cardiol 2004;44:1533-1542. 